\title{
Approximate Relations between Series and Integrals
}

\author{
By P. A. P. Moran
}

If we wish to evaluate numerically an integral of the form

$$
I=\int_{-\infty}^{\infty} f(x) d x
$$

we may replace it by the sum

$$
S=\sum_{h=-\infty}^{\infty} h f(n h+\delta) .
$$

Here $h$ is the interval width and $\delta$ a position constant. If $f(x)$ tends to zero suffciently rapidly at $x= \pm \infty$ and is reasonably continuous we may expect (2) to give a good approximation to (1). In practice it is often found that (2) is a much closer approximation to (1) than would have been guessed simply by looking at the graph of $f(x)$. For example if

$$
f(x)=(2 \pi)^{-1} e^{-1 x^{2}}
$$

and $h=2$ so that the 'panels' of the integration formula are very wide compared with the scale of variation of $f(x)$, we find that $S$, which is a periodic function of $\delta$, never diverges from $I=1$ by more than 0.015 whilst if $h=1$, the divergence is never greater than $0.52 \times 10^{-8}$ (Aitken [1], Yates [14]). On the other hand a function like $f(n)=\pi^{-1}\left(1+n^{2}\right)^{-1}$ gives a much lower accuracy $\left(0.37 \times 10^{-2}\right.$ for $\left.h=1\right)$.

If $f(n)$ tends to zero like $e^{-x^{2}}$, say, all its derivatives will also tend to zero at $\boldsymbol{x}= \pm \infty$, and use of the Euler-Maclaurin sum formula would suggest that $S=I$ exactly. The fallacy in the argument, explained by Goodwin [5], lies in the fact that the Euler-Maclaurin series is an asymptotic one only. Another reason for ex $\rightarrow$ pecting a good approximation arises from the Gregory formula for numerical integration (Whittaker and Robinson [13], p. 143) which expresses the integral as a sum of ordinates together with correction terms involving the differences at each end.

Another approach to this situation is provided by Poisson's summation formula (Fettis [4] and Salzer [11]). This is

$$
\int_{0}^{\infty} f(x) d x=h\left\{\frac{1}{2} f(0)+\sum_{k=1}^{\infty} f(k h)\right\}-2 \sum_{k=1}^{\infty} g\left(2 k \pi h^{-1}\right)
$$

where

$$
g(x)=\int_{0}^{\infty} f(t) \cos n t d t .
$$

When $f(x)$ is even it will often happen that $g(x)$ is very small for large $x$ and the approximation is then very good. Fettis, [4], gives a number of illustrations of the use of this fact.

Received November 15, 1956. 
This phenomenon of finding such surprising accuracy is not confined to integrals of functions which behave like $e^{-x^{2}}$. In a problem concerning dilution series of viruses (Moran [9]) it became necessary to sum the series

$$
\sum_{n=-\infty}^{\infty}\left(p^{3(2 n)}-p^{4\left(2^{n}\right)}\right)
$$

where $0<p<1$. This series is in fact periodic in $\delta$ where $p=\exp -2^{\delta}$. Its mean value for varying $\delta$ can easily be found to be

$$
\int_{-\infty}^{\infty}\left\{\exp \left[-3\left(2^{x}\right)\right]-\exp \left[-4\left(2^{x}\right)\right]\right\} d x=2-\log 3(\log 2)^{-1}=0.450375,
$$

and an actual exploration of the field shows that the sum never diverges from this value by more than 0.000004 .

Since any integral over a finite range can be turned into one over $(-\infty, \infty)$ this suggests a useful method of evaluating finite integrals. As an example consider the integral

$$
J=\int_{0}^{\infty} \frac{\exp -u^{2}}{X+u} d u
$$

which is tabulated by Goodwin and Staton [6]. For the value $X=0.2$ they give $J=1.5948$. Putting $u=\exp x^{3}$ we get

$$
J=3 \int_{-\infty}^{\infty} \frac{n^{2} \exp \left(-e^{2 x^{3}}+x^{3}\right.}{X+\exp x^{3}} d x .
$$

Taking intervals of width 0.5 in $n$ we get an estimate 1.58069 . A better method is to put $u=e^{x}$ so that

$$
J=\int_{-\infty}^{\infty} \frac{\exp \left(-e^{2 u}+u\right)}{X+e^{u}} d u
$$

and again taking intervals of width 0.5 we get an estimate 1.594812 whose accuracy is at least 0.0001 . One advantage of this method often is that when we want to evaluate an integral for a large number of values of some parameter in it (as $X$ in the Goodwin-Staton integral) we can do most of the calculation of the ordinates once and for all. A second advantage is that the accuracy increases extremely rapidly with decreasing $h$. It is also clear that the problem of deciding what particular transformation into an integral on the range $(-\infty, \infty)$ is best, is one which would repay more study.

The difficulty in using this technique in practice is to obtain an estimate of the error. One method is to calculate $S$ for $\delta=0,0.2, \cdots 0.8$, say (or $0.1,0.3, \cdots, 0.9$ ) and examine the variation by drawing a smooth curve. Since the mean value of $S$ is equal to $I, I$ must lie between the estimated largest and smallest value of the sum. The trouble with this is that it requires five to ten times as much work. However, it may be worth while to do this if the integral is required for many values of some parameter since it should be only necessary to estimate the error for two or three values of the parameter.

It may also, in some cases, be possible to obtain a still better estimate by the method invented by L. F. Richardson and called by him the 'deferred approach to 
the limit' (for some interesting examples of this see Salzer [12] and Bolton and Scoins [2]). We calculate the sum $S$ for a series of values of $h\left(h_{0}, 2 h_{0}, \cdots, n h_{0}\right.$ say) and use simple polynomial extrapolation to obtain an estimate for $h=0$. Some care is necessary in this. The remainder is a function not only of $h$ but also of the position of the grid. It could happen that an exact estimate was obtained for a particular value of $h$ and that for smaller $h$ the estimate moves away again from the true value. Moreover, the error may well be oscillatory. If, however, $f(n)$ is an even function and $\delta$ is put equal to zero it seems almost certain in most cases that the convergence will ultimately be monotonic and this method may be useful. It must also be remembered that in many cases the error will decrease faster than any power of $h$.

In some particular cases an estimate of the error can be calculated explicitly. This has been done in a valuable paper by Goodwin [5] who considers integrals of the form

$$
\int_{-\infty}^{\infty} \exp \left(-x^{2}\right) f(x) d x
$$

where $f(x)$ satisfies certain regularity conditions in the complex plane. In this case a good estimate for the error is, when $f(x)$ is taken as even, and $\delta=0$,

$$
E(h)=2 \exp \left(-\pi^{2} h^{-2}\right) \int_{-\infty}^{\infty} f\left(y-i \pi h^{-1}\right) \exp \left(-y^{2}\right) d y
$$

and if $f(n)$ does not increase too rapidly as $n$ tends to infinity this is approximately

$$
E(h) \div 2 \pi^{\frac{1}{2}} \exp \left(-\pi^{2} h^{-2}\right) f\left(i \pi h^{-1}\right) \text {. }
$$

This result with $f(x)=1$ can be used to evaluate more complicated integrals such as the integral of the multivariate normal distribution over the range

$$
\left(x_{1}>0, \cdots, x_{n}>0\right)
$$

when the correlations have certain prescribed forms (Moran [8]), and also certain incomplete integrals of the bivariate and trivariate normal distribution with arbitrary lower bounds (Das [3]).

The trouble with these methods when applied to other functions is to evaluate the error. If we have an even function then it is clear from (2) that $\partial S / \partial \delta$ is zero when $\delta=0$ and $\delta=\frac{1}{2} h$. Since $S$ is a periodic function it will usually happen that one of these is a maximum and the other a minimum. In fact we can expand $S$ in a Fourier cosine series and then it will usually be found that the first cosine term is much larger than all the rest. Thus we can be practically certain in most cases that if $f(n)$ is an even function its integral will lie between the limits

$$
\sum_{n=-\infty}^{\infty} h f(n h) \quad \text { and } \quad \sum_{n=-\infty}^{\infty} h f\left(\left(n+\frac{1}{2}\right) h\right) .
$$

This can be proved to be true for the integral (Das [3]).

$$
\frac{t \exp -\frac{1}{2} t^{2}}{2 \pi} \int_{-\infty}^{\infty} \frac{\exp -\frac{1}{2} x^{2}}{t^{2}+x^{2}} d x
$$


and since this is equal to

$$
(2 \pi)^{-\frac{1}{2}} \int_{t}^{\infty} \exp -\left(\frac{1}{2} x^{2}\right) d x
$$

Das has found two very sharp inequalities for the probability integral. However, if $w \epsilon$ add these sums together we get a vastly more accurate result for the same amount of labour.

It is also of some interest to notice that if $\delta$ is taken as a random variable with a uniform distribution on the interval $(0, h)$ the mean value of $S$ is the integral and its variance about this mean, like the error term above, can be found as a series in the Fourier transform of $f(x)$ (Moran [7]).

The above ideas can also be applied to integrals on the range $(0,2 \pi)$ of functions which are periodic with period $2 \pi$. In this case the sum over a finite number of points is often remarkably close to the integral. If, in addition $f(x)$ is an even function it is sometimes possible to obtain very sharp inequalities from above and below (Moran 10 ) for such functions as

$$
I_{0}=(2 \pi)^{-1} \int_{0}^{2 \pi} \exp (x \cos \theta) d \theta
$$

and this technique may be useful in calculating such functions as

$$
\int_{0}^{t} I_{0}(x) d x
$$

I am much indebted to a referee for some useful criticisms which have substantially improved this note.

The Australian National University,

$$
\text { P. A. P. Moran }
$$

\section{Canberra, A. C. T.}

1. A. C. Artken, Statistical Mathematics, Oliver and Boyd, Edinburgh, 1939.

2. H. C. Bolton \& H. I. Scorns, "Eigenvalues of differential equations by finite-difference methods," Camb. Phil. Soc., Proc., v. 52, 1956, p. 215-229.

3. S. C. DAs, "The numerical evaluation of a class of integrals, II," Camb. Phil. Soc., Proc., v. 52, 1956, p. 442-448.

4. H. E. FETTIS, "Numerical calculation of certain definite integrals by Poisson's summation formula," $M T A C$, v. 9, 1955 , p. 85-91.

5. E. T. Goodwin, "The evaluation of integrals of the form $\int_{-\infty}^{\infty} f(x) e^{-2} d x$," Camb. Phil. Soc., Proc., v. 45, 1949, p. 241-245.

6. E. T. Goodwin \& J. Staton, "Table of $\int_{0}^{\infty} \frac{e^{-u^{2}}}{u+x} d u$," Quart. J. Mech. Appl. Math., v. 1" 1948, p. 319-326.

7. P. A. P. Moran, "Numerical integration by systematic sampling," Camb. Phil. Soc., Proc., v. 46,1950 , p. $111-115$.

8. P. A. P. Moran, "The numerical evaluation of a class of in tegrals, I," Camb. Phil. Soc., Proc., v. 52, 1956, p. 230-233.

9. P. A. P. MORAN, "The dilution assay of viruses," J. Hygiene, v. 52, 1954, p. 189-193.

10. P. A. P. MORAN, "Inequalities for Bessel functions," Quart. J. Math. (to appear).

11. H. E. SALZER, "Aerial pattern synthesis; use of Poisson's formula," Wireless Engineer, v 33,1956, p. $240-244$.

12. Herbert E. SAlzer, "A simple method for summing certain slowly convergent series," J. Math. Phys., v. 33, 1954-55, p. 356-359.

13. E. T. Whi TTAKeR \& G. RoBinson, The Calculus of Observations, Blackie, London, 1932.

14. F. YAtes, "Systematic sampling," Roy. Soc. Phil., Trans., A, v. 241, 1948, p. 345-377. 